haltsanalytischen Instrumentarium, das unterschiedliche Grade von Verzerrungen - von „correct representations “ über „misinterpretations" bis hin $\mathrm{zu}$ "manipulations" (v. a. S. 165ff.) - zu erfassen versucht. Hier vermisst der kommunikationswissenschaftliche Leser Reliabilitätstests, und es bleibt der Verdacht, dass die valide und trennscharfe Codierung dieser Dimensionen weniger einfach ist, als es die klaren Befunde der Studie nahelegen. Dennoch aber verleiht die Einführung dieser wertenden Dimension der Analyse eine interessante Schärfe und Relevanz.

Auch darüber hinaus finden sich im Band eine Reihe von Perspektiven und Befunden, die inspirierend für Kommunikationswissenschaftler sein dürften, die sich für visuelle und/ oder für Klimawandel-Kommunikation interessieren. Dies trifft beispielsweise auf den Beitrag von Thomas Nocke (55ff.) zu, der - ohne die entsprechende Nomenklatur zu verwenden - die visuelle Seite der Mediatisierung von Wissenschaft beschreibt: Er untersucht, wie sich Datenvisualisierungen von einem eher der Außenkommunikation dienenden und für die wissenschaftliche Analyse selbst nahezu bedeutungslosen Hilfsmittel zu einem Kernbestandteil klimawissenschaftlicher Arbeit im Rahmen der „visual analytics“ (S. 62) entwickelt haben.

Diese wiederkehrenden anregenden Momente entschädigen denn auch dafür, dass andere Aspekte nur wenig oder aus kommunikationswissenschaftlicher Sicht in unbefriedigender Weise thematisiert werden. Dies gilt insbesondere für die Rezeption und die möglichen persuasiven Wirkungen der vorgestellten Bilder, die für den Gesamtband und viele seiner Einzelstudien letztlich zwar den Relevanz gebenden Fluchtpunkt darstellen, aber kaum wirklich untersucht werden.

Mike S. Schäfer

\section{Carsten Wünsch / Holger Schramm / Volker Gehrau / Helena Bilandzic (Hrsg.)}

Handbuch Medienrezeption

Baden-Baden: Nomos, 2014. - 450 S.

ISBN 978-3-8329-6610-2

Angesichts der Entwicklungen weg von der (rein) rezeptiven hin zur (auch) produktiven und - wenn man so will - partizipativen Mediennutzung mitsamt sich verwischender Grenzen stellt sich schon die Frage, ob ein „Handbuch Medienrezeption" nicht begrifflich aus einer Perspektive startet, aus der heraus das eigentlich Spannende aktueller Entwicklungen ausgesperrt bleibt. Im kurzen Vorwort wird Medienrezeption dann aber doch breit gefasst, „da es um Fragen und Phänomene geht, welche Rezipientinnen und Rezipienten betreffen und mit ihrem Kontakt mit Medieninhalten einhergehen" (S. 5). Den Kontakt sehen die drei Herausgeber und die Herausgeberin nicht begrenzt auf die eigentliche Nutzungssituation. Sie folgen vielmehr dem Verständnis der DGPuKFachgruppe „Rezeptions- und Wirkungsforschung", die "neben Prozessen der Wahrnehmung, Verarbeitung und Interpretation von Medienangeboten auch Fragen der Zuwendung und der Auswahl von Medien(-inhalten) und deren Aneignung und Integration in die je eigene Lebenswelt als relevant erachtet“ (ebd.).

In zentralen Punkten spiegelt sich dieses ganzheitliche Verständnis auch in der Gesamtschau der 26, insgesamt 400 Seiten umfassenden Beiträge zu den aktuellen Perspektiven und Forschungsfeldern der Rezeptionsforschung wider. In vier Kapiteln werden die Bereiche Grundlagen der Rezeption, Selektion und Zuwendung, Phänomene und Erlebniswelten und Kontexte der Rezeption näher beleuchtet. Mit Blick auf den Anspruch, der an ein Handbuch zu stellen ist, gefallen die Beiträge mit einer guten Lesbarkeit, oft auch dann, wenn sie in dichtes Terrain vorstoßen. Sie führen zunächst prägnant ins Thema ein, entfalten dieses meist klar strukturiert und in der gebotenen Differenziertheit und bringen das Wesentliche am Ende noch einmal auf den Punkt.

Wenn man den Menschen verstehen will, muss man sich sein Denken, Fühlen und Handeln ansehen! Die ersten drei Beiträge in Kap. 1 folgen diesem Primat und entfalten die Themen Kognition, Emotion und Handlung komprimiert und treffsicher in ihrer besonderen Bedeutung für Rezeptionsprozesse. Dies gelingt in aller Regel, ohne einer zu hohen Informationsdichte zu erliegen, und mit nützlichen Verweisen auf die anderen Beiträge im Handbuch, die das kurz Angesprochene dann differenzierter betrachten. Die Grundlagen der Rezeption werden im vierten und letzten Beitrag noch um die wichtige Ebene der Wahrnehmung und Verarbeitung ergänzt. Unter dem Label Kanalspezifika wird hier auf zentrale Unterschiede des Sehens, Hörens und Lesens von Informationen fokussiert.

In Kap. 2 umreißen sechs Beiträge gelungen das Spektrum der Prozesse, die der eigentlichen Rezeption (idealtypisch) vorgelagert sind. Begrifflich mit Diffusion und Domestizierung eingefangen werden zunächst die Prozesse der Verbreitung und Aneignung (neuer) Medien „zusammen gedacht“, eine Makro- und Mikro- 
perspektive zueinander gebracht. Nachfolgender Beitrag zu Alltag und Routinen nimmt einen zentralen Hintergrund für Medienrezeption gesondert in den Blick und gefällt durch seine gelungene Auswahl der skizzierten Perspektiven „auf die subjektive Bedeutung des Mediengebrauchs im Kontext der alltäglichen Lebensführung" (S. 110). Es folgt das Thema Einstellung, das vor allem in seiner besonderen Bedeutung für die selektive Zuwendung zu Medieninhalten betrachtet wird.

Als einführende Handbuchartikel gefallen auch die letzten drei Beiträge in Kap. 2. Zunächst wird beim Thema Persönlicbkeit anschaulich gezeigt, „dass Persönlichkeitsmerkmale - unter Kontrolle trivialer Faktoren wie Soziodemographie - regelmäßig einen beachtlichen Teil der Varianz von Medienhandlungen erklären“ (S. 138). Nachfolgender Beitrag zu Motivation verharrt inhaltlich glücklicherweise nicht in der Darstellung von Motivkatalogen, sondern skizziert Modelle und Ansätze, in denen auch die handlungstheoretische Sichtweise eine Berücksichtigung erfährt. Der abschließende Beitrag Stimmung führt in die bekannte Mood-Management-Theorie ein und referiert die Bedeutung für Medienselektion fest im Blick - theoretische Weiterentwicklungen und alternative Ansätze.

Die neun Beiträge in Kap. 3 sind ausgewählten Phänomenen und Erlebniswelten der Medienrezeption gewidmet. Hier gefällt, dass neben Themen wie Urteilsbildung und Bewertung, Verstehen, Emotionales Erleben, Spannung und Immersion auch die Bereiche Empathie und Identifikation, Ästhetisches Erleben, Parasoziale Interaktion und sozialer Vergleich eine angemessene Würdigung erfahren. Der Beitrag Wabrgenommener Realismus wendet sich den auflösenden Grenzen von Realität und Fiktion zu und geht u. a. der Frage nach, ,wie Rezipientinnen und Rezipienten mit medienvermittelten Realitätsindikatoren umgehen" (S. 291). Der Fokus liegt auf grundsätzlichen kommunikationswissenschaftlichen Perspekti- ven, weniger auf sich aktuell aufdrängende Themen z. B. des Reality-TV.

Sinnvoll abgerundet wird das Gesamtthema des Handbuchs in Kap. 4 mit sieben Beiträgen zu den Rezeptionskontexten. Zuerst erfolgt ein Zugang zur Mediennutzung in den Lebensphasen. Dieser erscheint beim Lesen zu reduziert auf die beiden Phasen Adoleszenz und Seniorenalter und bei den referierten Studien nicht ganz auf dem Stand. Die folgenden Beiträge widmen sich der Rezeption in der Gruppe, Bedeutung von physischen und psychischen Einschränkungen sowie der Rezeption im öffentlichen Raum, die mit den aktuellen Medienentwicklungen eine zunehmend wichtige Perspektive auf Rezeptionsprozesse repräsentiert.

Mit den letzten drei Beiträgen wird das Thema des Handbuchs um weitere Perspektiven bereichert. Die Bearbeitungen der Themen Vergemeinschaftung und Epoche integrieren auch die wichtige Perspektive der (zunehmenden) Mediatisierung kommunikativen Handelns, der angesichts ihrer besonderen Bedeutung im aktuellen Fachdiskurs ein eigenständiger Beitrag durchaus zugestanden hätte. Der mit $\mathrm{Kul}$ tur überschriebene Beitrag überführt anschaulich dargestellt den Gegenstandsbereich der Rezeptionsforschung in eine interkulturell vergleichende Perspektive.

Die sich daran anschließende, klar strukturierte, auf die Bedingungen, Praktiken und Bedeutung von Mediennutzung fokussierte Darstellung ist eine „Blaupause“ für die Bearbeitung eines Themas in einem Handbuch, das sich an ein breites „Fachpublikum aus Kommunikationswissenschaft und angrenzenden Disziplinen $[\ldots]$ richtet sowie an Studierende im fortgeschrittenen Bachelorstudium und im Masterstudium “ (S. 5). Gerade Studierende dürfen sich auf ein Grundlagenwerk freuen, das ihnen ein breites Spektrum von Prozessen, Phänomenen und Kontexten der Medienrezeption nahe bringt, ohne nur an der Oberfläche zu kratzen. Daniel Hajok 